Editorial

Z Herz- Thorax- Gefäßchir 2019 · 33:229-230 https://doi.org/10.1007/s00398-019-0321-7

(c) Springer Medizin Verlag $\mathrm{GmbH}$, ein Teil von Springer Nature 2019

Wie gelangen wir zu Erkenntnissen über Zusammenhänge in den Naturwissenschaften, zu denen selbstverständlich auch die Lebenswissenschaften zu zählen sind? Die Frage, was können wir wissen, ist Jahrtausende alt und wurde im wissenschaftlichen Sinn abseits mythologisch geprägter Vorstellungen mit hoher Wahrscheinlichkeit erstmals durch die vorsokratischen griechischen Philosophen gestellt.

Die Erkenntnisphilosophie unterscheidet zwei Prinzipien der Aufdeckung naturwissenschaftlicher Zusammenhänge, Empirismus und Rationalismus. In Umrissen skizziert, bedeutet Empirismus, aus Erfahrungen und Beobachtungen einzelner Zusammenhänge im Experiment durch Induktion auf übergeordnete Prinzipien rückzuschließen; Rationalismus, aus einer zumindest logischen Prinzipien gehorchenden, erdachten, vermeintlich widerspruchsfreien Theorie mittels Deduktion einzelne Zusammenhänge abzuleiten und deren Gültigkeit im Experiment zu überprüfen. Hauptsächlich auf Empirie begründende Erkenntnistheorien entwickelten Bacon, Locke, Berkeley und Hume. Die frühen Hauptvertreter des Rationalismus, die die grundsätzliche Idee einer allgemein gültigen Vorherrschaft der Vernunft in ihren Schriften behandelten, sind Descartes, Leibniz, Wolff und Spinoza. Es verwundert nicht, dass sich in ihren Reihen bedeutende Mathematiker fanden.

In der modernen klinischen Medizin entsprechen Therapieentscheidungen und deren Begründung vermehrt nach Kriterien des Empirismus den Ergebnissen kontrollierter randomisierter Studien und Untersuchungen. Dieser Heran-

\author{
A. Welz \\ Halblech, Deutschland
}

\title{
Was können wir wissen?
}

gehensweise kommt zweifelsohne große Berechtigung zu. Allerdings entstammen die daraus gezogenen Schlussfolgerungen meist selektionierten Patientengruppen. In der Behandlung des Einzelfalls und individuellen Patienten kann es somit durchaus ebenso berechtigt sein, therapeutische Maßnahmen quasi deduktiv aus gültigen allgemeinen naturwissenschaftlichen Gesetzen abzuleiten.

Dies sei beispielhaft an der Kontroverse restriktiver vs. liberaler Transfusionsregime erläutert. So folgt die Hämodynamik allgemeinen physikalischen Gesetzen. Bezieht man zudem die GesetzmäBigkeiten der Rheologie sowie die Beziehungen von Herzminutenvolumen und $\mathrm{Hb}$ (Hämoglobin)-Gehalt des Bluts zur Sauerstofftransportkapazität in die Berechnungen mit ein, lässt sich ein unter dem Gesichtspunkt maximale Sauerstoffversorgung des Myokards bei zugleich möglichst ökonomischer Herzarbeit optimaler Hb-Wert errechnen. Dieser ist bei einem Wert von circa $12 \mathrm{mg}$-\% anzusetzen. Insoweit bleibt hier hinterfragbar, ob die aus randomisierten Studien mit Einschluss meist nichtherzchirurgischer $\mathrm{Pa}$ tienten abgeleitete Empfehlung einer restriktiven Transfusionstherapie mit Tolerierung von $\mathrm{Hb}$-Werten um $8 \mathrm{mg} / \%$ oder darunter gerade bei kritisch kranken Koronarpatienten zur Anwendung gebracht werden soll.

Liebe Leserinnen und Leser, ich habe mir diesen knappen Ausflug in die Philosophie der Naturwissenschaften erlaubt, da die vorliegende Ausgabe unserer Zeitschrift für Herz-, Thorax- und Gefäßchirurgie in der Rubrik „Evidenzbasierte Medizin“ einen ausführlichen Artikel von A. Böning zur auf den ersten Blick provo- kativen Frage: „Register oder randomisierte Studien. Was bringt die Wahrheit?“ beinhaltet. Der Autor zeigt hier an publizierten Beispielen klar auf, dass Erkenntnisgewinn und Fortschritt in der Medizin auf mehrere Standbeine gegründet sein sollten. Darüber hinaus müssen alle getroffenen Aussagen und daraus abgeleiteten Behandlungsempfehlungen kritisch hinterfragt und geprüft werden.

Bereits Karl Popper forderte im frühen 20. Jh. für jede ernsthafte wissenschaftliche Theorie oder Hypothese deren prinzipielle Falsifizierbarkeit.

Dies könnte gerade für die chirurgische Therapie der Aortenklappenstenose und die derzeit noch gültigen Leitlinien bedeutsam werden. Derzeit weist die Mehrzahl der Studien, randomisiert wie registerbasiert, auf eine Überlegenheit der Transkathetertechnik in einem weiten Indikationsfeld hin. Auch gibt es derzeit keine Hinweise auf ein frühes Implantatversagen. Somit muss in diesem Fall, basierend auf Empirie, die heute gültige Indikationsstellung zum offenen Aortenklappenersatz bei Kranken mit niedrigem Risiko möglicherweise in naher Zukunft hinterfragt werden.

Die übrigen Autoren der weiteren Rubriken mögen mir die Fokussierung dieses Editorials auf das Problem von Erkenntnis und Wahrheit verzeihen. Die vorliegende Ausgabe enthält lehrreiche und an den aktuellen Stand der Wissenschaft anknüpfende Arbeiten zu operativen Techniken ebenso wie aktuelle biologische, grundlagenwissenschaftliche Themen.

Ich wünsche Ihnen, liebe Leserinnen und Leser, ebenso viel Freude, Genuss wie Erkenntnisgewinn bei Lesen und 
Studium der vorliegenden Ausgabe der Zeitschrift Herz-, Thorax- und Gefäßchirurgie.

Ihr

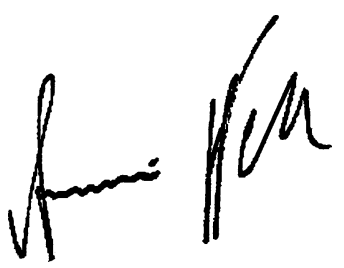

A. Welz

\section{Korrespondenzadresse}

Prof. Dr. A. Welz
Jägerweg 2,87642 Halblech,
Deutschland
armin.welz@uni-bonn.de

Interessenkonflikt. A. Welz gibt an, dass kein Interessenkonflikt besteht.

\section{Unterschätzte Kontaminationsquelle: Computertastaturen}

Die Tastaturen und Computermäuse in Kliniken und Arztpraxen sind meist stark mit Erregern belastet - und nicht wenige mit multiresistenten Keimen.

Von vielen Personen genutzte Kontaktflächen wie Türgriffe sind die idealen Verbreitungswege für nosokomiale Infektionen. Auch Tablets und Computerperipheriegeräte wie Tastaturen und Mäuse zählen dazu. Die einzelnen Geräte werden zwar nur von einem limitierten Personenkreis genutzt, dafür ist der Kontakt mit Fingern und Händen umso intensiver. Ärzte von der Universität in Seattle haben anhand einer Literaturrecherche geschaut, wie häufig über Kontaminationen solcher Geräte in Kliniken und Praxen berichtet wird. Sie fanden insgesamt $75 \mathrm{Stu}$ dien, von denen sich 50 auf eine Analyse der Keime beschränkten, in 25 Studien wurde auch der Nutzen von Desinfektionen geprüft. Insgesamt war in den Untersuchungen die Kontamination von 2800 Geräten, hauptsächlich Tastaturen, getestet worden.

\section{Hohe Kontaminationsrate}

Irgendwelche Keime fanden sich in fast allen Studien an fast allen untersuchten Geräten, die Kontaminationsrate betrug im Schnitt 97\%. In einer Studie auf Intensivstationen waren immerhin nur 24\% der Geräte kontaminiert. Zumeist handelte es sich um wenig gefährliche Hautbewohner, doch wurden nicht selten auch potentielle Pathogene gefunden, am häufigsten Staphylococcus aureus. Diesen Keim fanden die Forscher im Schnitt bei $28 \%$ aller Geräte; die Bandbreite variierte je nach Studie aber beträchtlich zwischen $1 \%$ und $94 \%$.

MRSA, Clostridium und Co.

Methicillin-resistente Staphylokokken

(MRSA) wurden im Schnitt in 14\% der Proben nachgewiesen, bei einer vergleichbaren Bandbreite: In einigen Kliniken und Praxen waren also fast alle untersuchten Tastaturen und Mäuse MRSA-kontaminiert, in vielen dagegen keine. Vancomycin-resistente Keime entdeckten die Forscher im Schnitt bei knapp $4 \%$ aller Geräte ( $0 \%$ bis $12 \%)$, Clostridium difficile bei $8 \%$ (0\% bis $28 \%$ ).
Von den 25 experimentellen Studien zur Desinfektion berichten 14 über eine signifikante Reduktion der Keimzahl durch diverse Mittel wie Isopropanol, Chlorhexidin oder UV-Licht. In den übrigen 9 Studien gelang dies nicht oder die Forscher konnten die Resultate nicht klar interpretieren.

\section{Desinfektionsprogramme wirksam?}

In 5 der Studien wurde geprüft, ob spezielle Desinfektionsprogramme die Kontamination und Infektion von Ärzten, Pflegekräften sowie Patienten reduzieren. In 3 der Studien gingen mit solchen Programmen Infektionsraten von MRSA und anderen pathogenen Keimen zurück, allerdings sei fraglich, ob es hier einen kausalen Zusammenhang gebe, so die Studienautoren.

Unterm Strich zeigten die Studien, dass fast alle Tastaturen und Mäuse in Kliniken und Praxen mit Keimen kontaminiert seien, nicht wenige auch mit Pathogenen. Wie relevant solche Kontaminationen für die Sicherheit von Patienten sind, sei jedoch unklar. Auch welche Desinfektionsmethoden den größten Erfolg versprechen, lasse sich nicht sagen, dazu seien die Studien zu heterogen, geben die Forscher zu bedenken. Letztlich könnten Tastaturen und Mäuse aber eine bedeutsame und unterschätzte Kontaminations- oder gar Infektionsquelle darstellen, heißt es in dem Beitrag.

Quelle: Ärzte Zeitung basierend auf: BMJ Open (2019)

9:e026437 